\title{
MODELING AIDS VACCINES: THE CELLULAR LEVEL
}

\author{
CLAUDIO J. STRUCHINER \& M. ELIZABETH HALLORAN*
}

GRIS, Université de Montréal 2375 Ch. Côte Ste-Catherine Montréal, QC Canada H3T 1A8*Division of Biostatistics, Emory University School of Public Health 1599 Clifton Road, N. E. Atlanta, GA USA 30329

This paper discusses current strategies for the development of AIDS vaccines which allow immunization to disturb the natural course of HIV at different detailed stages of its life cycle. Mathematical models describing the main biological phenomena (i.e. virus and vaccine induced $T 4$ cell growth; virus and vaccine induced activation of latently infected T4 cells; incremental changes in immune response as infection progresses; antibody dependent enhancement and neutralization of infection) and allowing for different vaccination strategies serve as a background for computer simulations. The mathematical models reproduce updated information on the behavior of immune cells, antibody concentrations and free viruses. The results point to some controversial outcomes of an AIDS vaccine such as an early increase in virus concentration among vaccinated when compared to nonvaccinated individuals.

Key words: AIDS vaccines - vaccine models

Considerable effort is now being directed toward the development of AIDS vaccines (Cohen, 1990). Initial speculations about the development and testing of these vaccines point to plausible beneficial as well as undesirable outcomes of vaccination programs at the individual and population levels. At the individual level, AIDS vaccines may enhance disease, induce immune suppression, show antibodymediated enhancement of virus infection (Levy, 1988), and promote neurologic dysfunction (Fauci, 1988). Altematively, a vaccine might benefit the vaccinated individual by slowing or preventing development of disease without protecting against becoming infected. This could result in either an increase or a decrease in the duration and degree of infectiousness of the vaccinated person for other individuals. The mechanisms of proposed AIDS vaccine candidates (Koff \& Hoth, 1988) might, therefore, change transmission, resulting in possibly unexpected and undesirable indirect effects at the population level.

Clinical manifestations of AIDS are attributed to a disruption of the equilibrium among

This investigation was supported by a Rockefeller Foundation grant to the AIDS and Reproductive Health Network, a Ford Foundation grant to IMS/UERJ and the Brazilian Research Council (CNPq).

Received 23 August 1991.

Accepted 20 December 1991. the various cells and their roles in the immune system and subsequent attack by opportunistic infections (Levy, 1989). HIV-1 infection is associated with depletion of the CD4 subset of T lymphocytes, which is also the primary receptor for HIV binding. The relative density and integrity of each component of the immune system play an important role in the determination of the clinical aspects and level of infectiousness of infected individuals. Recent advances in the molecular biology of HIV make it possible that vaccine development strategies focus on specific components or cellular interactions of the immune system network. As a consequence, the description of parameters important for tracing the progression of the disease in the population must also reflect changes at the cellular level for each epidemiological category of risk.

On the other hand, vaccination strategies must take into consideration characteristics that affect the population as a whole, such as prevaccination baseline immunity, resistance and prevalence of infection as well as the dominant modes of transmission. The outcome of a vaccination program among a population still largely uninfected might differ from one in a population with a high level of transmission and prevalence. Moreover, different risk groups contribute distinctively to the overall transmission pattern and might require different levels 
of attention. Two major patterns of transmission have been described. The first one contains a strong heterosexual component and is observed in African countries. Alternatively, in Europe and North America transmission among homosexuals and intravenous drug users dominate the pattern. We can speculate that in some areas, such as Brazil, where blood transfusion has been implicated as an important mode of transmission, a third major epidemiological pattern may emerge. Different vaccines might be more effective in risk groups with different modes of transmission, depending on their exact mechanism of action at the individual and cellular level.

Transmission of virus between individuals can be either cell-free or cell-associated (Bolognesi, 1989a). Within individuals, transmission can also be by free virus or cell-tocell contact. Current strategies for the development of AIDS vaccines are based on various approaches that allow immunization to disturb the natural course of the HIV at different detailed stages of its life cycle. Vaccines targeting specific inter- or intra- person transmission mechanisms might have different epidemiological consequences for morbidity patterns. Therefore we can talk about different AIDS vaccines with different epidemiological characteristics.

We present in this paper a formulation for an AIDS vaccine model where transition rates among compartments are described as a function of the density of specific cells of the immune system. This approach, which takes place at the cellular level, is the first step toward a population model in which transition rates also reflect relevant characteristics of each epidemiological category of risk, such as sexual behavior. Such a population model would provide the basic structure for discussing demographic consequences of an AIDS vaccination program. As an example, such a model would allow us to explore the different consequences of vaccines delivered indiscriminately to the whole population as compared to strategies targeting specific risk groups. When formulated at the population level, an AIDS vaccine model will help understanding important methodological issues in the evaluation and testing of AIDS vaccines.

In the next section, we present the model of the interaction of the virus and the vaccine antigen with the immune system. In the third section, we present the initial numerical studies of the effect of vaccination on the course of HIV infection. In the discussion, we delineate the consequences of these results and directions for further exploration.

\section{MODEL}

The deterministic, compartmental model developed by Perelson (1989) describing the interaction of the immune system with HIV contains many of the aspects required for the study of AIDS vaccine mechanisms. Perelson's model is based on the life history of HIV and reproduces certain features of AIDS such as "the long latent period, the almost complete absence of free virus particles, the low frequency of infected T4 cells and the slow T4 cell depletion seen during the course of the disease" (Perelson, 1989, p. 350). While his model is constructed with a discussion of the basic biological puzzles faced by AIDS researcher in mind, the equations are intentionally general. This allows description of the potential biological phenomena produced by various vaccine candidates or phenomena predicted by the various vaccine development strategies.

In what follows, we describe each of our compartments and corresponding equations making explicit the modifications we introduced to the general model described in (Perelson, 1989). Compartments represented in this system are T4 cells that can be susceptible $(T)$, latently infected $\left(T^{*}\right)$, or actively infected $\left(T^{* *}\right)$, free virus (v), vaccine antigen (vac), syncytia $(S)$, neutralizing antibody $\left(A_{n}\right)$ and enhancing antibody $\left(A_{e}\right)$.

Susceptible T cells - The dynamics of the susceptible T4 cell compartment are described by:

$$
\begin{aligned}
& \frac{d l}{d l}=s^{-r^{\prime}}-\mu_{T} T+r T\left(\frac{1}{\left.1+t^{\prime} V_{v}-p_{\mathrm{v}} r^{\prime}\right)}\right) \times\left(1-\frac{T+T^{*}}{T_{\max }}\right)+
\end{aligned}
$$

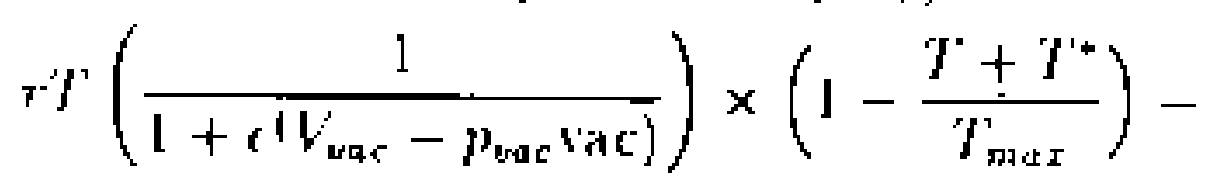

$$
\begin{aligned}
& k_{1} \cdot T\left(1+\frac{1}{1+c^{t} A_{a b}-A_{e}}\right)-k_{s} T \cdot \cdots-k_{a} T s
\end{aligned}
$$

$T$ denotes the total concentration of uninfected T4 cells which are produced according to the first term in equation $1, s e^{-\gamma \nu}$, wheres $s$ is the rate of supply of new T 4 cells. This rate is diminished by the total free virus load (v) by the factor $e^{-\gamma \nu}$. T4 cells die at a rate $\mu_{T}$ which is estimated as the inverse of the lifetime of uninfected cells. 
The third term in equation 1 accounts for virus-induced $\mathrm{T} 4$ cell stimulation and proliferation. This rate is denoted by $r$. When virus is absent, the term

$$
\frac{1}{1+e\left(V_{v}-p_{v} v\right)}
$$

is approximately zero, since $V_{v}$ is large positive, and no activation takes place. When virus load is high compared to $\mathrm{V}_{v}$, this term approaches 1 . Then the $\mathrm{T} 4$ cells proliferate proportionally to $r$. The virus-induced stimulation is also modulated by the total amount of susceptible $(T)$ and infected $\left(T^{*}\right)$ T4 cells. As the concentration of these cells approaches $\mathrm{T}_{\max }$ virus-induced $\mathrm{T} 4$ cell stimulation slows. The term

$$
1-\frac{T+T^{*}}{T_{\max }}
$$

varies between 0 and $1 . \mathrm{T}_{\max } p_{\mathrm{v}}$ and $V_{\mathrm{v}}$ are parameters that regulate these behaviors.

Vaccine antigen (vac) can also stimulate susceptible T4 cell growth in a manner analogous to that of the virus antigen. The T4 cell growth rate is multiplied by

$$
\frac{1}{1+e\left(V_{v a c}-p_{v a c} \mathrm{VaC}\right)}
$$

which approaches zero, when the concentration of vaccine antigen is low, and 1 otherwise. Expression of the growth rate is also affected by the total concentration of susceptible and latently infected T4 cells as previously described. $V_{v a c}$ and $P_{v a c}$ are regulating parameters analogous to $V_{v}$ and $p_{v}$.

Viruses infect T4 susceptible cells according to the rate $k_{1}$. Both virus and vaccine antigens can induce antibodies $A_{e}$ that enhance the infection rate (Bolognesi, 1989b). The factor

$$
1+\frac{1}{1+e^{\left(A_{a b}-A_{e}\right)}},
$$

which assumes values in the interval $(1,2)$, accounts for this by increasing from 1 to 2 as the concentration of antibodies that enhance the infection rate increases. $A_{a b}$ is the regulating parameter for this process.

Two additional mechanisms account for the depletion of susceptible T4 cells. Syncytia can form between susceptible T4 cells and actively infected $\mathrm{T}$ cells $\left(T^{* *}\right)$ proportional to the rate $k_{s}$. Already existent syncytia can also adhere susceptible T4 cells directly proportional to the rate $k_{s}^{\prime}$. These mechanisms are represented by the last two terms in equation 1.
Latently infected T4 cells - Equation 2 describes the dynamics of latently infected $T$ cells $\left(T^{*}\right)$ :

$$
\begin{aligned}
\frac{d T^{*}}{d t}= & k_{1} v T\left(1+\frac{1}{1+e^{\left(A_{a b}-A_{e}\right)}}\right)-\mu_{T} T^{*}- \\
& k_{2}\left(1+\frac{1}{\left.1+e^{\left(V_{1}-\left(l_{v} v+l_{v a c} \mathrm{vac}\right)\right)}\right) T^{*}-}\right. \\
& k_{s} T^{*} T^{* *}-k_{s}^{\prime} T^{*} S
\end{aligned}
$$

$T^{*}$ are produced by the infection of susceptible $\mathrm{T} 4$ cells by virus as described above for equation 1. Latently infected T4 cells die at the same rate $(\mu \mathrm{T})$ as susceptible $\mathrm{T} 4$ cells.

Latently infected T4 cells naturally progress to actively infected cells at a rate $k_{2}$. Antigen (virus or vaccine) activation increases the rate or progression by a factor dependent on the total concentration of antigens. This process is represented by the expression

$$
1+\frac{1}{1+e\left(V_{l}-\left(l_{v} v+l_{v a c} \mathrm{Vac}\right)\right)}
$$

which increases from 1 to 2 as the total concentration of antigen increases. The activation of latent $\mathrm{T} 4$ cells by viruses could be represented by a greater factor. $V_{p} l_{v}$ and $l_{\nu a c}$ are the regulating parameters for this process.

Latent $T^{*}$ cells are also depleted by syncytia formation with $T^{* *}$ cells proportional to the rate constant $k_{s}$ and by binding directly to syncytia (S) at a rate proportional to the constant $k^{\prime}$.

Actively infected T4 cells $-T^{* *}$ are the viru-producing $\mathrm{T} 4$ cells obtained from the activation of latently infected cells. This is described by the first term in equation 3 . Activated $T^{* *}$ cells die at the rate $\mu_{b}$ and the contingent of cells that participate in syncytia formation are also depleted from this compartment at a rate $k_{s}$

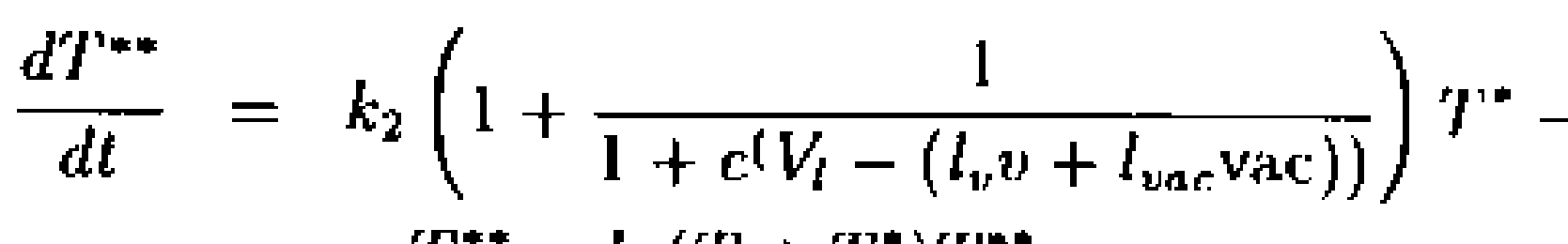

$$
\begin{aligned}
& \mu_{b} T^{* *}-k_{s}\left(T+T^{*}\right) T^{* * *}
\end{aligned}
$$

Free viruses - Viruses are produced from actively infected T4 cells and syncytia when these cells die at rates $N \mu_{b}$ and $N_{g} \mu_{s}$ respectively. Viruses are depleted by binding to T4 susceptible cells i. l.

$$
k_{1} v T\left(1+\frac{1}{1+e\left(A_{a b}-A_{e}\right)}\right),
$$


natural death $\left(\mu_{w}\right)$ and antibody neutralization $\left(k_{n} v A_{n}\right)$.

$$
\begin{aligned}
\frac{d v}{d t}= & N_{\mu_{b}} T^{* *}+N_{,} \mu_{s} S- \\
& k_{1} v T\left(1+\frac{1}{1+e^{\left(A_{a b}-A_{e}\right)}}\right)- \\
& \mu_{v} v-k_{n} v A_{n}
\end{aligned}
$$

A summary of the activities of virus and vaccine antigens is given in Table $I$.

\section{TABLE I}

Viral and vaccine antigen activities

\begin{tabular}{lcc}
\hline Activity & $\begin{array}{c}\text { Viral } \\
\text { parameter }\end{array}$ & $\begin{array}{c}\text { Vaccine antigen } \\
\text { parameter }\end{array}$ \\
\hline $\begin{array}{l}\text { Infection-enhancing } \\
\text { antibodies }\end{array}$ & $s_{e}^{v}$ & $s_{e}^{v a c}$ \\
$\begin{array}{l}\text { Neutralizing } \\
\text { antibodies }\end{array}$ & $s_{n}^{v}, k_{n}$ & $s_{n}^{v a c}$ \\
$\begin{array}{l}\text { T4 cell } \\
\text { proliferation } \\
\text { Latent cell } \\
\text { activation }\end{array}$ & $p_{v}, V_{v}$ & $p_{v a c}, V_{v a c}$ \\
$\begin{array}{l}\text { Inhibition of } \\
\text { T4 cell growth }\end{array}$ & $l_{v}, V_{l}$ & $l_{v a c}, V_{l}$ \\
Infection of & $e^{-\gamma}$ & \\
T4 cells & & n.a. \\
\hline
\end{tabular}

Vaccine antigens - The possible actions of the vaccine antigens on the immune system have been designed to mimic those of the viral antigen with the exception that vaccine antigens cannot infect T4 cells or inhibit T4 cell growth. By setting the parameters for the vaccine equal to those of the virus, the antigen behaves similarly to the virus in those aspects. Specific behaviors corresponding to subunit vaccines can be simulated by setting certain vaccine antigen parameters to zero.

Once injected in the individual, vaccine antigens show an exponential decay according to equation 5 .

$$
\frac{d \mathrm{vac}}{d t}=-\mu_{\mathrm{a}} \mathrm{vac}
$$

Syncytia - Actively infected cells can fuse with other cells to form multinucleated giant cells called syncytia. The expression below describes the dynamics of this compartment, which contains terms described above in addition to the term describing syncytia death $\left(\mu_{s}\right)$. The dynamics is the same as proposed in (Perelson, 1989).

$$
\frac{d S}{d t}=k_{s}\left(T+T^{*}\right) T^{* *}-\mu_{s} S
$$

Neutralizing Antibodies - The humoral immune response to the HIV challenge could include production of antibodies that neutralize the virus, as well as antibodies that enhance infection of susceptible T4 cells. Neutralizing antibodies are expected to block cell infection by HIV in opposition to the antibodies that enhance the rate of cell infection. Descriptions of possible mechanisms of the opposing action of these two antibody types are found in (Bolognesi, 1989a).

Equation 7 describes the dynamics of the neutralizing antibody compartment. Neutralizing antibody is produced at a rate proportional to the total concentration of antigens $\left(s^{v}{ }_{n} v+\right.$ $\left.s_{n}^{v a c} \mathrm{vac}\right)$ and the number of T4 susceptible cells (T). The proportionality parameters are $s_{n}{ }_{n}$ and $s_{n}{ }^{v a c}$. Antibody production ceases as its concentration reaches a maximum $\left(A_{n \max }\right)$. The remaining two terms account for antibody decay $\left(\mu_{a} A_{n}\right)$ and antibody consumed when binding to virus $k_{n} v A_{n}$

$$
\begin{aligned}
\frac{d A_{n}}{d t}= & \left(s_{n}^{v} v+s_{n}^{v a c} \mathrm{vac}\right) T\left(1-\frac{A_{n}}{A_{n \max }}\right) \\
& -\mu_{a} A_{n}-k_{n} v A_{n}
\end{aligned}
$$

Enhancing Antibodies - Equation 8 is analogous to equation 7 in that it describes the dynamics of the enhancing antibody compartment. In the absence of these antibodies, HIV infects T4 susceptible cells at a rate $k_{I} \nu T$. when the enhancing activity is present an extra amount of infection occurs at a rate

$$
k_{1} v T\left(\frac{1}{1+e\left(A_{a b}-A_{e}\right)}\right)
$$

This is also the rate at which this antibody type is consumed when performing the enhancing activity. 


$$
\begin{aligned}
\frac{d A_{e}}{d t}= & \left(s_{e}^{v} v+s_{e}^{v a c} \mathrm{vac}\right) T\left(1-\frac{A_{e}}{A_{e m a x}}\right)-\mu_{a} A_{e}- \\
& k_{1} v T\left(\frac{1}{\left.1+e^{(} A_{a b}-A_{e}\right)}\right)
\end{aligned}
$$

\section{RESULTS}

The numerical integration of the system of differential equations 1-8 was done using SimGauss (Ford, 1988), a simulation package written in the Gauss language (Aptech, 1988) for the MS DOS type computer environment. The variable step size integration algorithm chosen was the 2nd/3rd order Runge-KuttaFehlberg which requires three derivatives evaluations and is 3rd order accurate. With this algorithm it is possible to control for two error conditions, maximum and relative, which define the integration truncation error for each state variable at each step, which in turn set the variable step size. Stability of the results were verified by changing the integration algorithms, the integration steps and error constants in SimGauss and also by performing key simulations in two other packages, Dynamical Software I and II (Schaffer, 1988) and Mathematica (Wolfram, 1988).

Baseline equilibrium - The equilibrium value $\left(5.0 \times 10^{9}\right)$ reached by the T4 susceptible cells compartment $(T)$ in the absence of virus is approximately the same as the one found in (Perelson, 1989). The initial value for $T$ is 100 and zero for all other state variables. Parameter values are as in Table II. Since we introduced several other parameters in equations $1-8$, the meaning of the old parameters has also changed. Instead of reexpressing the dimensions of old parameters we kept the same absolute value as in (Perelson, 1989) for the sake of comparability but represent our time scale in arbitrary units.

Since $V_{v}$ and $V_{v a c}$ are large positive numbers, all terms in equation 1 are zero or nearly zero in the absence of virus and vaccine. Thus the equilibrium value of the susceptible T4 cells is determined solely by the rate of constant influx from the bone marrow and the death rate:

$$
\begin{aligned}
0= & s-\mu_{T} T_{0}+r T_{0}\left(\frac{1}{1+e^{V_{v}}}\right) \times\left(1-\frac{T_{0}}{T_{\max }}\right)+ \\
& r T_{0}\left(\frac{1}{1+e^{V_{\text {vac }}}}\right) \times\left(1-\frac{T_{0}}{T_{\max }}\right) \\
T_{0} \approx & \frac{s}{\mu_{T}} .
\end{aligned}
$$

For the parameter values in Table II this gives a predicted value of $T_{\mathrm{o}}=5.0 \times 10^{9}$, which agrees with the numerical results.

TABLE IJ

Valũes for model parameters

\begin{tabular}{clcl} 
Parameter & Value used & Parameter & Value used \\
\hline$s$ & $1.5 \mathrm{E}+07$ & $\gamma$ & $1.0 \mathrm{E}-05$ \\
$\mu_{T}$ & 0.003 & $r$ & $4.5 \mathrm{E}-03$ \\
$T_{\max }$ & $1.5 \mathrm{E}+10$ & $k_{\mathrm{t}}$ & $1.0 \mathrm{E}-10$ \\
$A_{\text {max }}$ & $1.0 \mathrm{E}+02$ & $A_{a b}$ & $1.0 \mathrm{E}+02$ \\
$k_{s}$ & $1.0 \mathrm{E}-11$ & $k_{s}^{\prime}$ & $1.0 \mathrm{E}-11$ \\
$k_{2}$ & $5.0 \mathrm{E}-06$ & $\mu_{b}$ & 0.05 \\
$\mu_{s}$ & 0.03 & $\mu_{v}$ & 0.1 \\
$N$ & 750 & $N_{s}$ & 850 \\
$k_{n}$ & $1.0 \mathrm{E}+13$ & $s_{v}$ & $1.5 \mathrm{E}-10$ \\
$s_{n}^{v}$ & $1.5 \mathrm{E}-10$ & $s_{n}^{v a c}$ & $1.5 \mathrm{E}-10$ \\
$s_{e}^{v}$ & $1.5 \mathrm{E}-10$ & $s_{e}^{\text {vac }}$ & $1.5 \mathrm{E}-10$ \\
$A_{\text {emax }}$ & $1.0 \mathrm{E}-13$ & $A_{\text {nmax }}$ & $1.0 \mathrm{E}-13$ \\
$V_{l}$ & $1.0 \mathrm{H}-02$ & $\mu_{a}$ & 0.03 \\
$V_{v}$ & $1.0 \mathrm{E}-02$ & $V_{v a c}$ & $1.0 \mathrm{E}-02$ \\
$p_{v}$ & 1.0 & $p_{v a c}$ & 1.0 \\
$l_{v}$ & 1.0 & $l_{\text {vac }}$ & 1.0 \\
& & & \\
\hline
\end{tabular}

If $V_{v}$ and $V_{v a c}$ were large negative numbers, and in the absence of virus and vaccine, the equilibrium solution for the susceptible $\mathrm{T} 4$ cells would be similar to that of Perelson, if $r$ in our model is one half the value of $r$ in Perelson's model. This can be shown by solving for the equilibrium value of susceptible T4 cells, $T_{0}$, from equation 1 in the absence of virus and vaccine. Let $r$ ' and $r$ ' the parameters sensitive to virus and vaccine antigen, respectively. Then at equilibrium, assuming $V_{v}$ and $V_{v a c}$ are large negative numbers,

$$
\begin{aligned}
0= & s-\mu_{T} T_{0}+r^{\prime} T_{0}\left(\frac{1}{1+e^{V_{x}}}\right) \times\left(1-\frac{T_{0}}{T_{\max }}\right)+ \\
& r^{\prime \prime} T_{0}\left(\frac{1}{1+e^{V_{v a x}}}\right) \times\left(1-\frac{T_{0}}{T_{\max }}\right) \\
T_{0} \approx & \frac{T_{\max }}{2}\left(1-\frac{\mu_{r}}{r^{\prime}+r^{\prime \prime}}+\sqrt{\left(1-\frac{\mu_{T}}{r^{\prime}+r^{\prime \prime}}\right)^{2}+\frac{1 s}{\left(r^{\prime}+r^{\prime \prime}\right) T_{\max }}}\right)
\end{aligned}
$$

If $r^{\prime}=r^{\prime \prime}$ then it can be replaced by $r$, so that $r^{\prime}+r^{\prime \prime}=2 r$. This is similar to the solution of Perelson's equilibrium value of susceptible $\mathrm{T} 4$ cells in the absence of virus.

Virus and vaccine challenges occurring separately - When the individual is challenged with a virus inoculation, the number of susceptible T4 cells increases to a level of $1.0 \mathrm{x}$ 
T4 susceptible cells

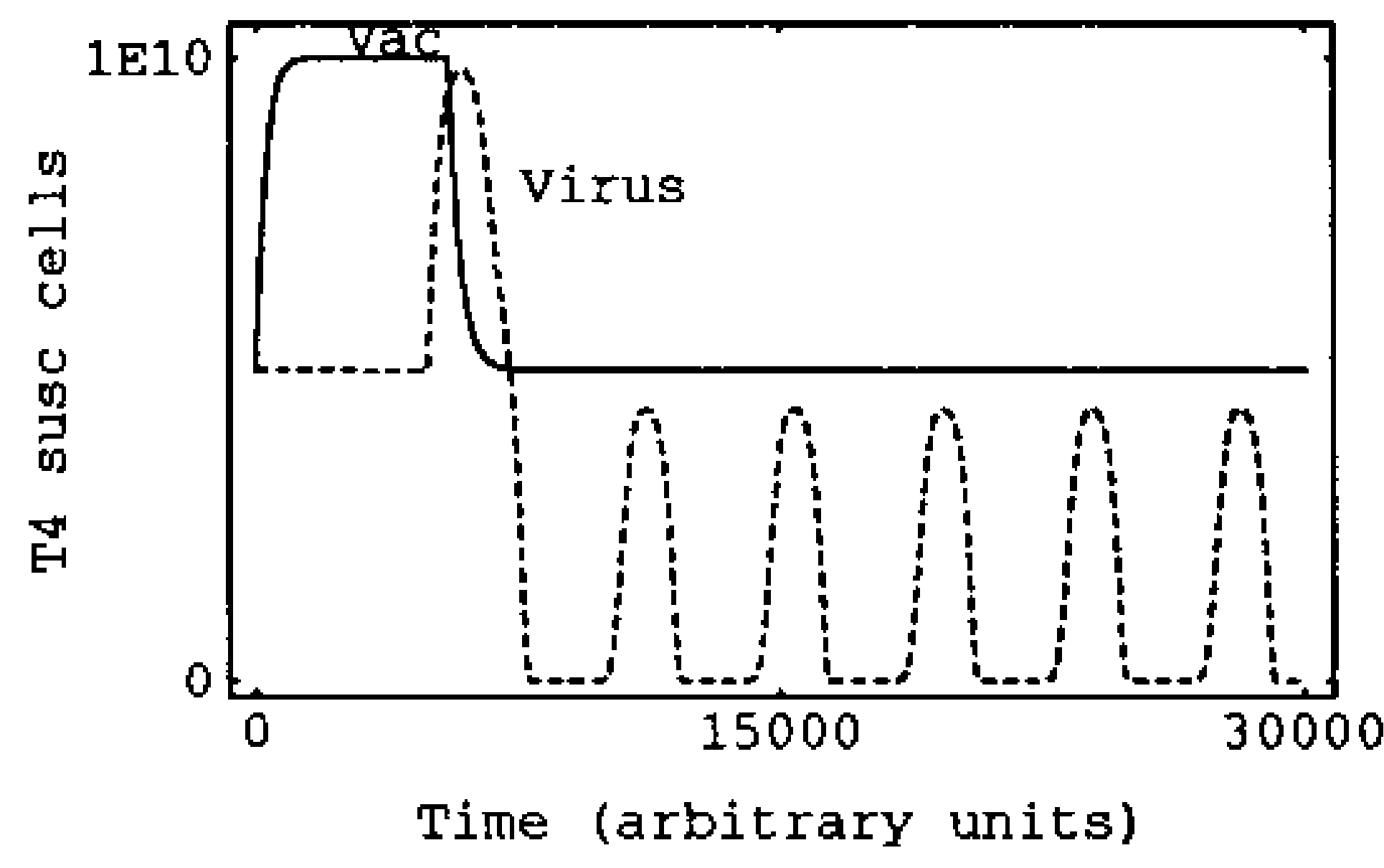

Fig. 1: T4 susceptible cells dynamics under virus and vaccine inoculation. Virus inoculation occurs at time 1,500 with 1,500 virus particles. Vaccine inoculation occurs at time zero with a concentration of $1.5 \times 10^{9}$ arbitrary units. Simulation parameters are as in Table II and both challenges occur separarely. $T_{0}=5.0 \times 10^{9}$.

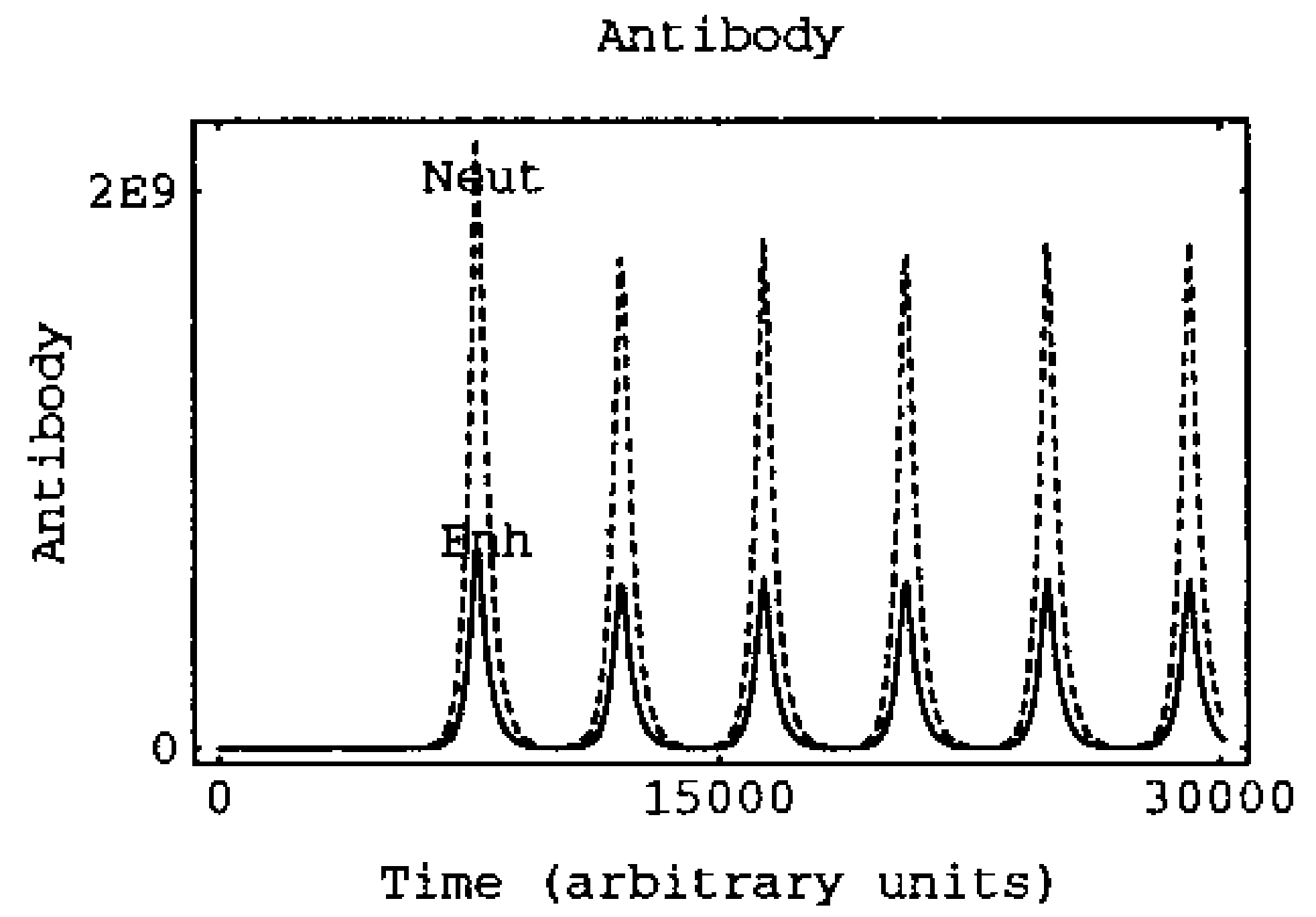

Fig. 2: comparison of neutralizing and enhancing antibody dynamics when induced by a vinus antigen. In both cases virus inoculum was 1,500 particles and occurred at time 1,500. Parameter values and initial values as in previous figure.

$10^{10}$ cells (Fig. 1). Once the T4 cells begin to decrease, the population shows intense, irregular oscillatory behavior. The susceptible T4 cell population can reach extreme values as low as 20 cells (not shown in the figure). Fig. 1 also shows the behavior of the susceptible T4 cells when challenged with a vaccine antigen that stimulates the immune system similarly to the viral antigen. It induces both infection-enhancing and neutralizing antibodies, as well as promotes $\mathrm{T} 4$ cell proliferation and activates latently infected cells.

Concentration levels achieved by enhancing and neutralizing antibodies in response to the virus are shown in Fig. 2. The same virus concentration and inoculation time was used in both simulations. The behavior of both antibody types are in synchrony but neutralizing antibodies reach a higher concentration level with the parameters used in the simulation.

The concentration of neutralizing antibodies oscillates in synchrony with the number of viruses (Fig. 7) when these are the single stimulus to antibody production. On the other hand, vaccine antigens alone induce neutralizing antibodies that decay as the vaccine antigen decays. This behavior is the same for both types of vaccines, neutralizing and enhancing.

The concentration of enhancing antibodies also oscillates in synchrony with the number of viruses (Fig. 7) when these are the single stimulus to antibody production. The dynamics of enhancing antibodies when induced by the vaccine antigen follows the same course as the other antibody type.

T4 susc cells behavior

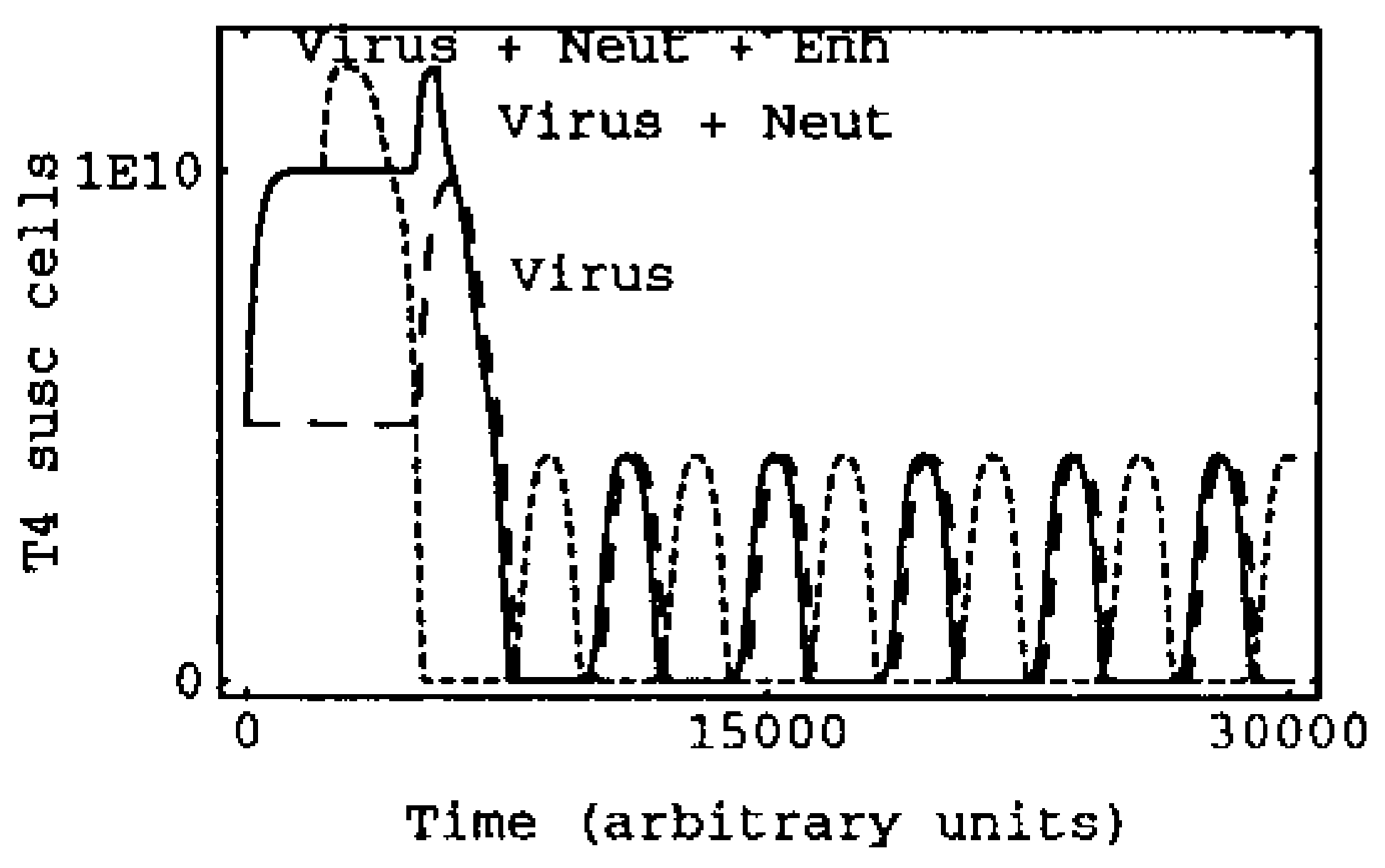

Fig. 3: T4 susceptible cells behavior when challenged with neutraljzing and enhancing vaccine antigen (Neut + Enh) and virus antigen (Neut + Enh + Virus). Vaccine antigen was inoculated at time zero and virus at time 1,500 . The virus inoculum was 1,500 particles. Parameter values and initial values as in previous figures.

Virus and vaccine challenges occurring together - Virus and vaccine antigens can either be inoculated together or delayed by any time interval. Fig. 3 summarizes susceptible T 4 cells behavior when challenged with both virus antigen (a virus inoculum of 1,500 particles at time 1,500$)$ and a vaccine antigen that induces neutralizing antibodies (Virus + Neut), virus plus a vaccine antigen that induces both neutralizing and enhancing antibodies (Virus + Neut + Enh) and virus antigen alone (Virus). The neutralizing vaccine induces neutralizing antibodies and stimulates susceptible $\mathrm{T} 4$ cell 
T4 lat cells behavior

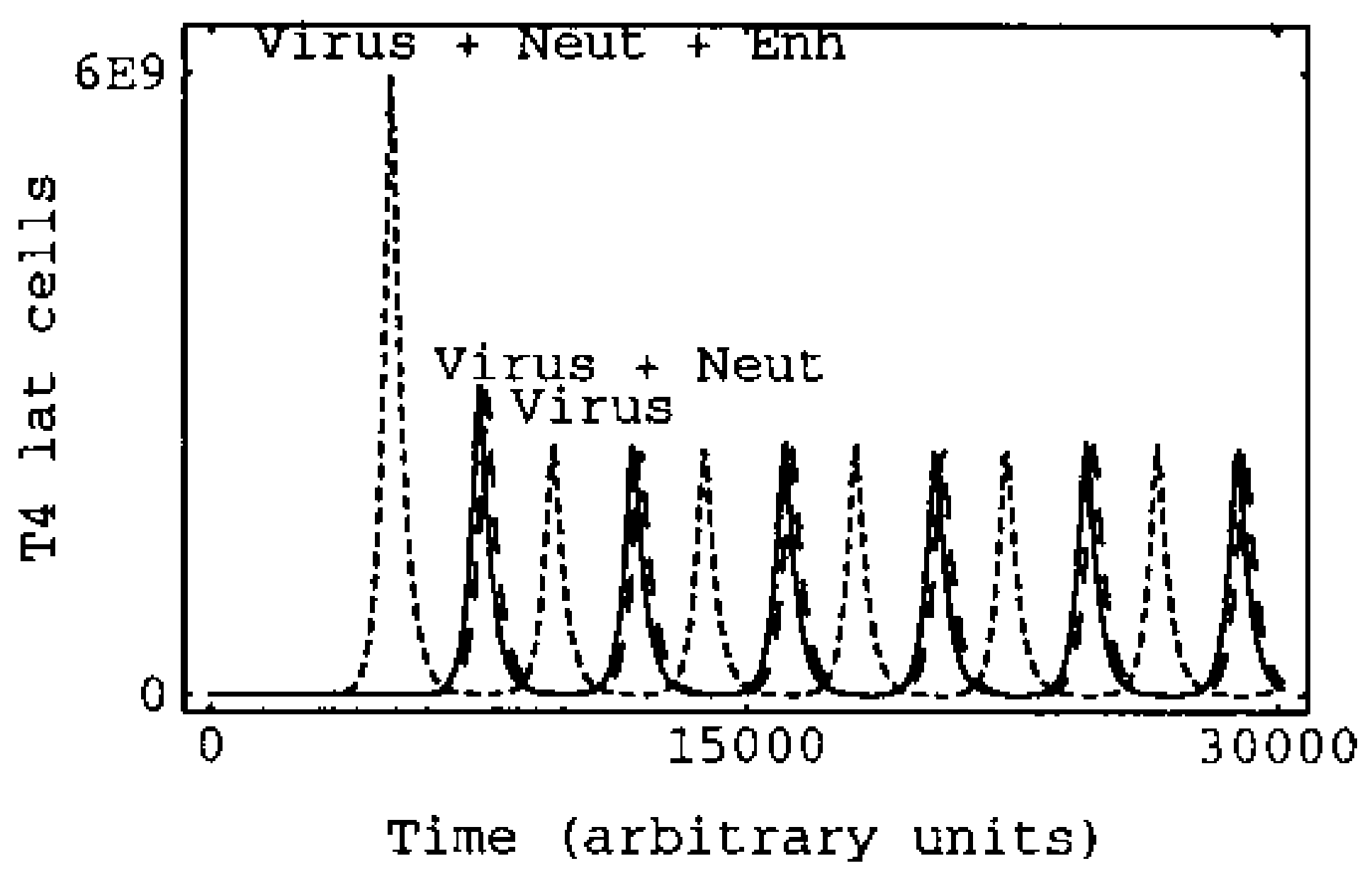

Fig. 4: latently infected T4 cells behavior when challenged with virus antigen and neutralizing vaccine antigen (Virus + Neut), neutralizing and enhancing vaccine antigens (Virus + Neut + Enh) and virus antigen alone (Virus). Vaccine antigen was inoculated at time zero and vinus at time 1,500. The vinus inoculum was 1,500 particles. Parameter values and initial values as in previous figures.

T4 act cells behavior

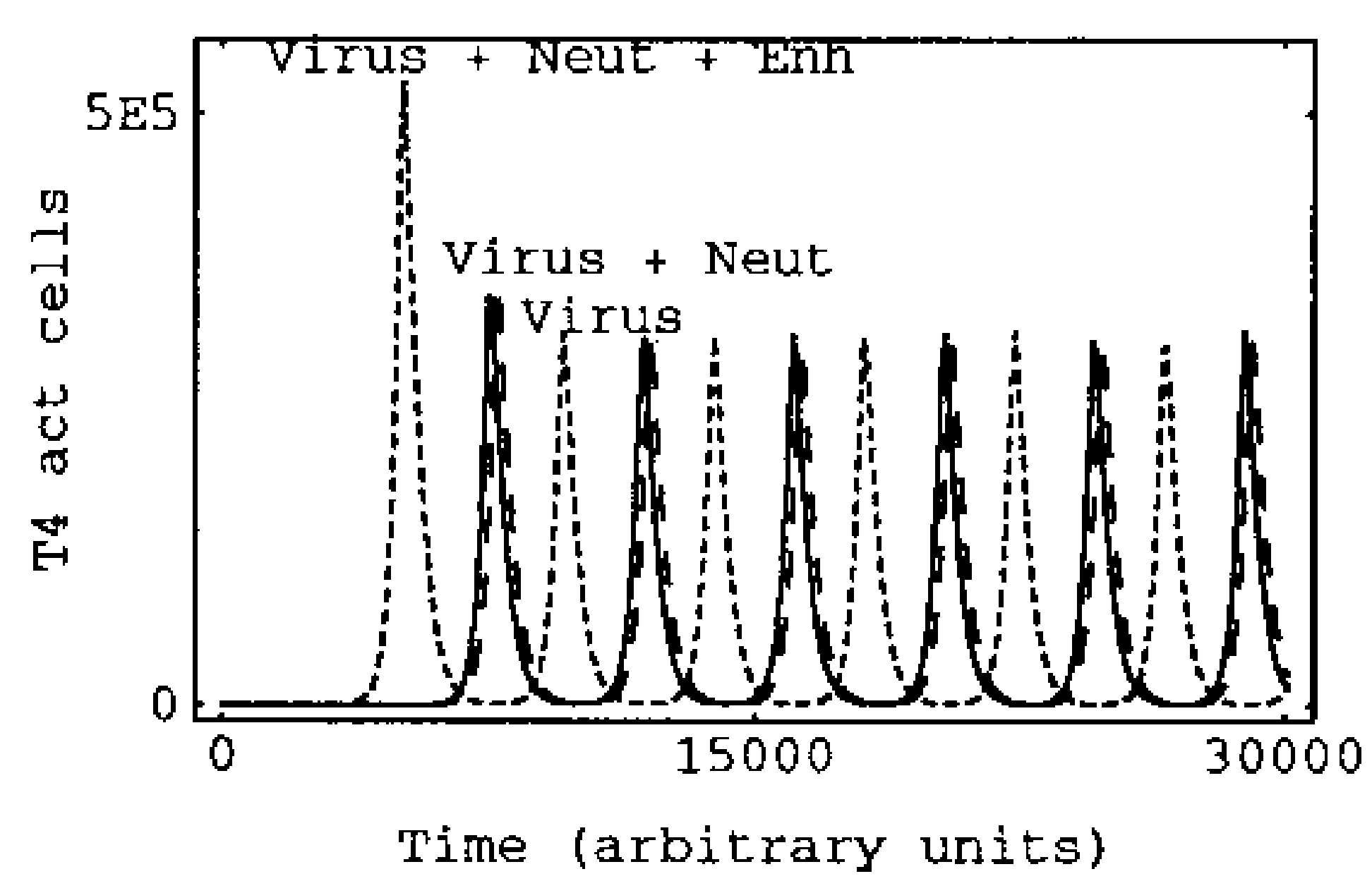

Fig. 5: actively infected T4 cells bahavior when challenged with virus antigen and neutralizing vaccine antigen (Virus + Neut), neutralizing and enhancing vaccine antigens (Virus + Neut + Enh) and virus antigen alone (Virus). Vaccine antigen was inoculated at time zero and virus at time 1,500. The virus inoculum was 1,500 particles. Parameter values and initial values as in previous figures.

production, but does not activate latently infected T4 cells or induce enhancing antibodies. The enhancing and neutralizing vaccine induces both antibody types as well as stimulates susceptible T4 cell proliferation and activates latently infected T4 cells. Vaccination with the enhancing antigen speeds up the course of the virus infection in the initial stages. These patterns can be compared with Fig. 1 when the antigens are inoculated one at a time.

Figs 4, 5 and 6 are analogous to Fig. 3 and show now the behavior of latently and actively

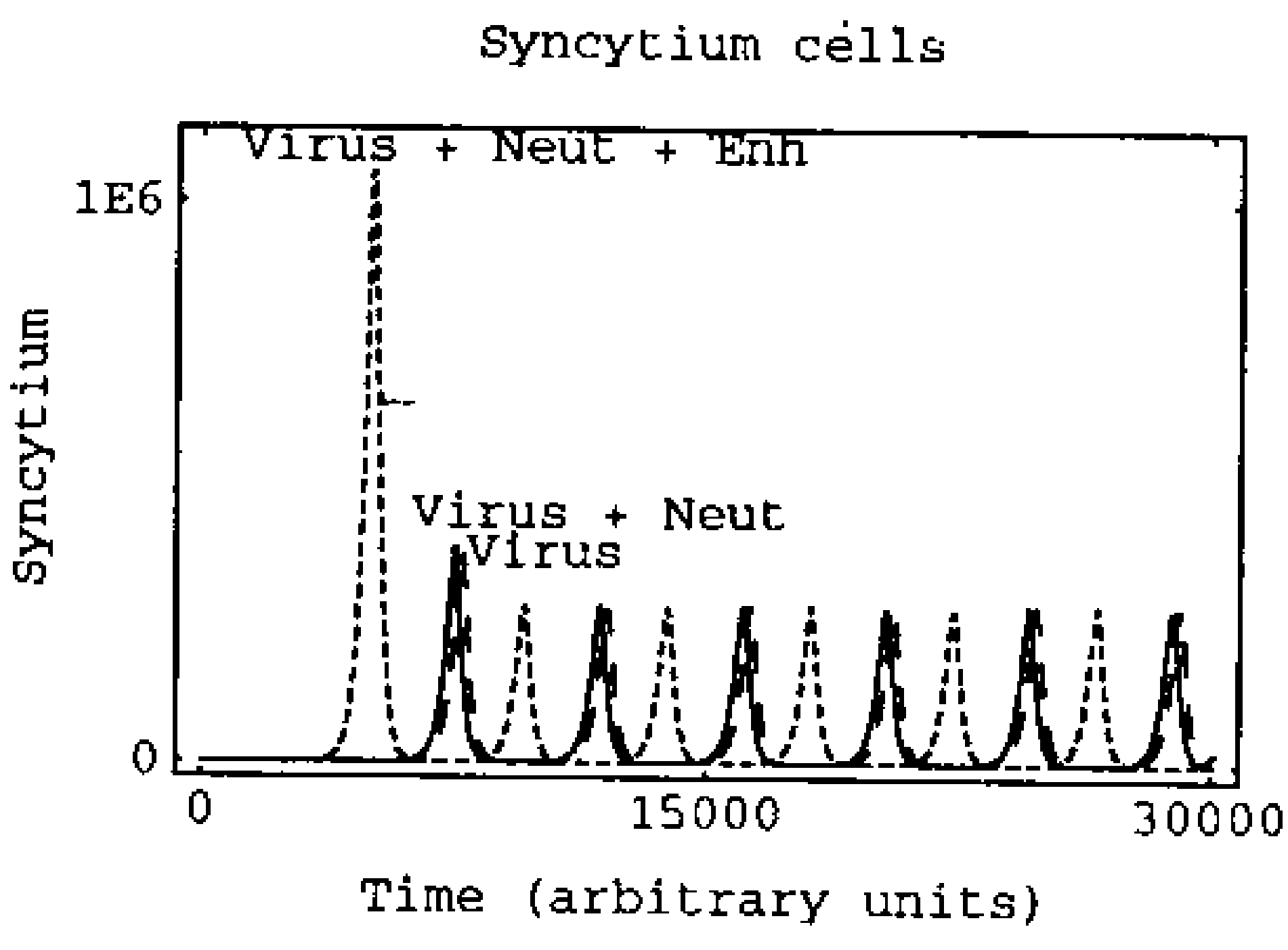

Fig. 6: syncytium compartment behavior when challenged with virus antigen and neutralizing vaccine antigen (Virus + Neut), neutralizing and enhancing vaccine antigens (Virus + Neut + Enh) and virus antigen alone (Virus). Vaccine antigen was inoculated at time zero and virus at time 1,500. The virus inoculum was 1,500 particles. Parameter values and initial values as in previous figures.

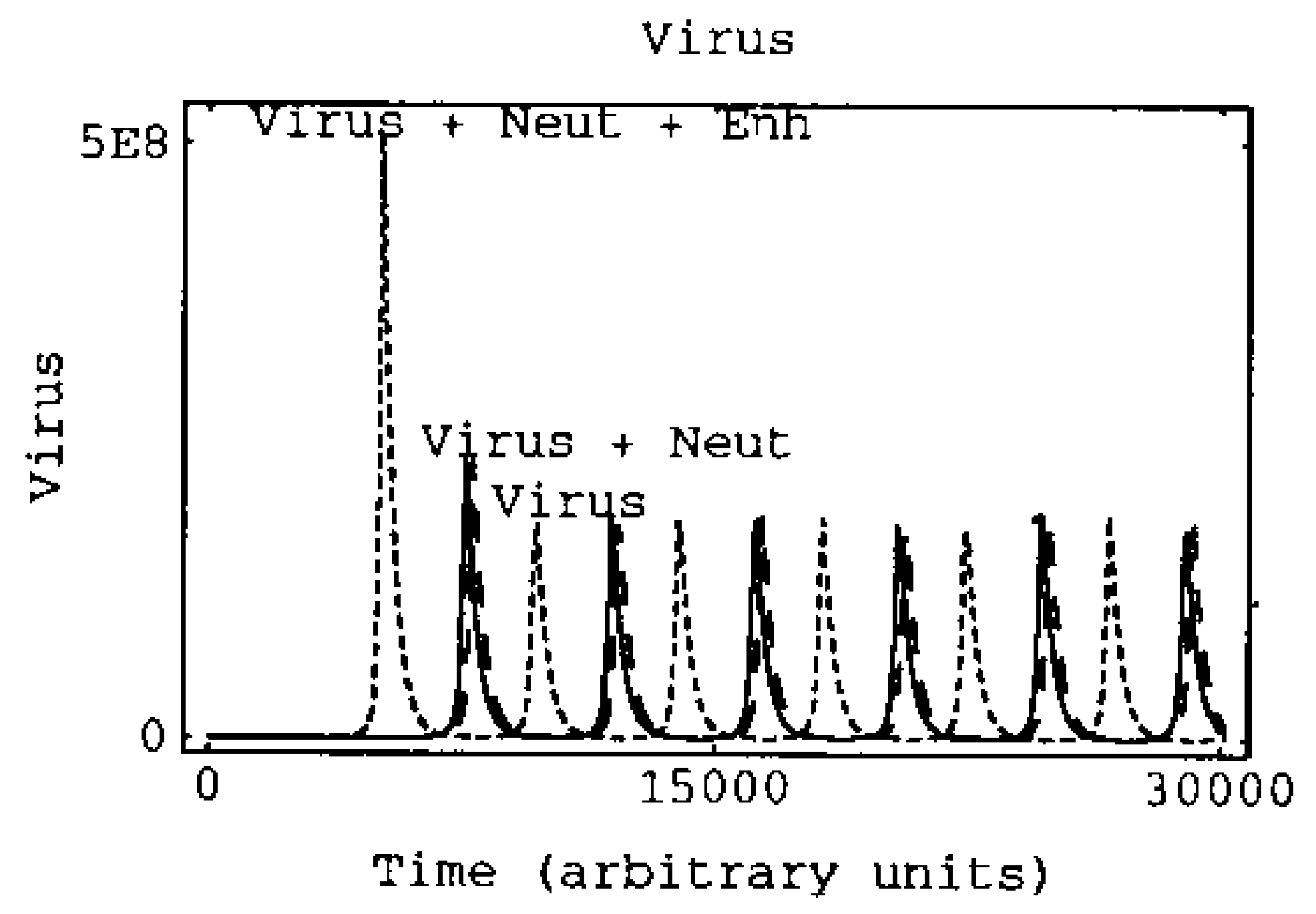

Fig. 7: establishment of virus infection after inoculation of virus antigen and neutralizing vaccine antigen (Virus + Neut), neutralizing and enhancing vaccine antigens (Virus + Neut + Enh) and virus antigen alone (Virus). Vaccine antigen was inoculated at time zero and virus at time 1,500. The virus inoculum was 1,500 particles. Parameter values and initial values as in previous figures.

infected T4 cells as well as syncytia. The concentrations of susceptible and latently infected T4 cells are out of phase with each other. When virus is inoculated in an individual who was previously vaccinated with an antigen that induces both neutralizing and enhancing antibodies, virus infection establishes earlier, and the number of virus particles is initially higher. The presence of neutralizing antibodies causes little changes in the natural course of infection paradoxically advancing the establishment of infection for a few time units. This behavior, seen in Fig. 7, also determines the pattern shown in previous figure. 


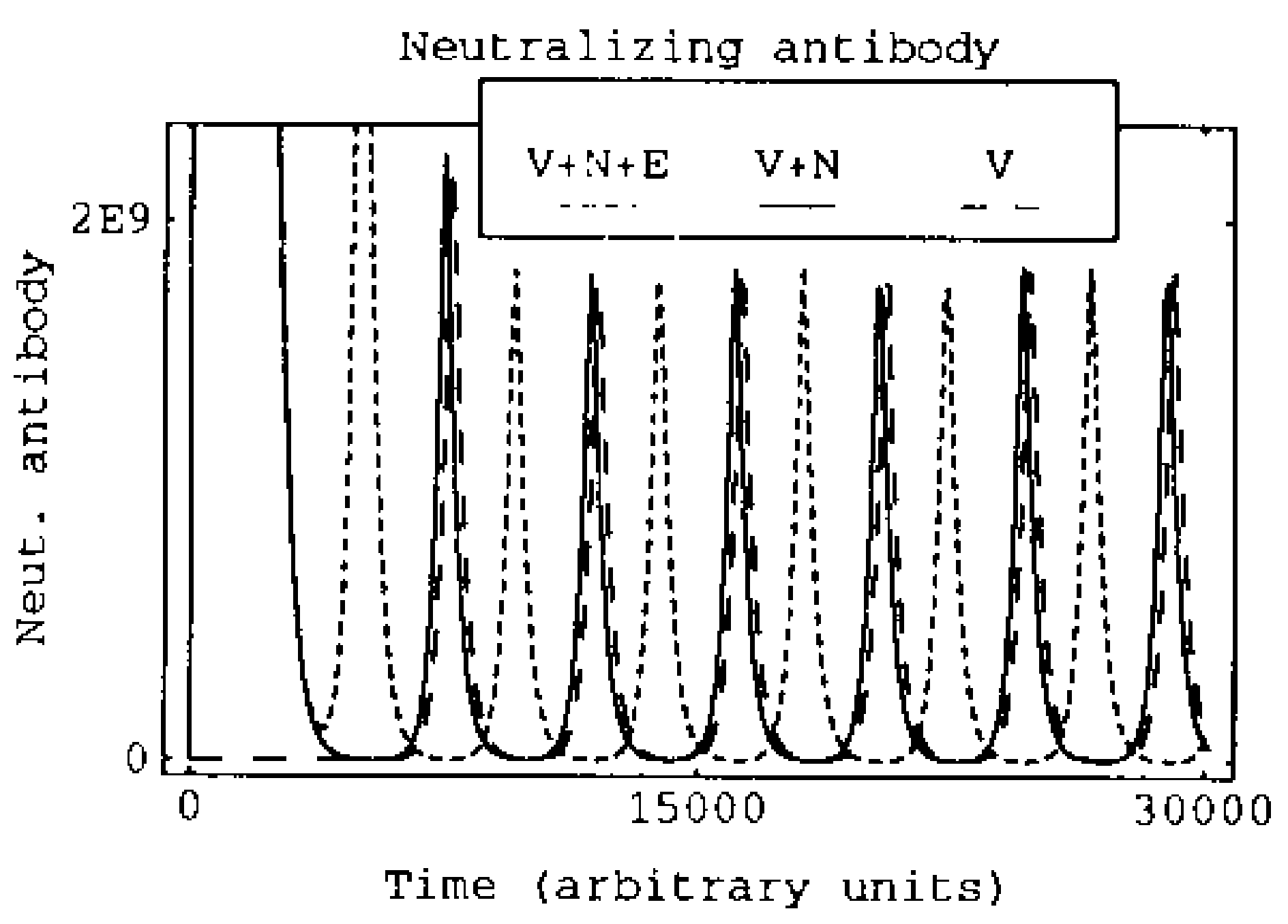

Fig. 8: neutralizing antibody behavior after inoculation of virus antigen and neutralizing vaccine antigen $(\mathrm{V}+$ $\mathrm{N}$ ), neutralizing and enhancing vaccine antigens ( $\mathrm{V}+\mathrm{N}$ $+\mathrm{E})$ and virus antigen alone (V). Vaccine antigen was inoculated at time zero and virus at time 1,500. The virus inoculum was 1,500 particles. Parameter values and initial values as in previous figures. Extreme values are not shown in figure.

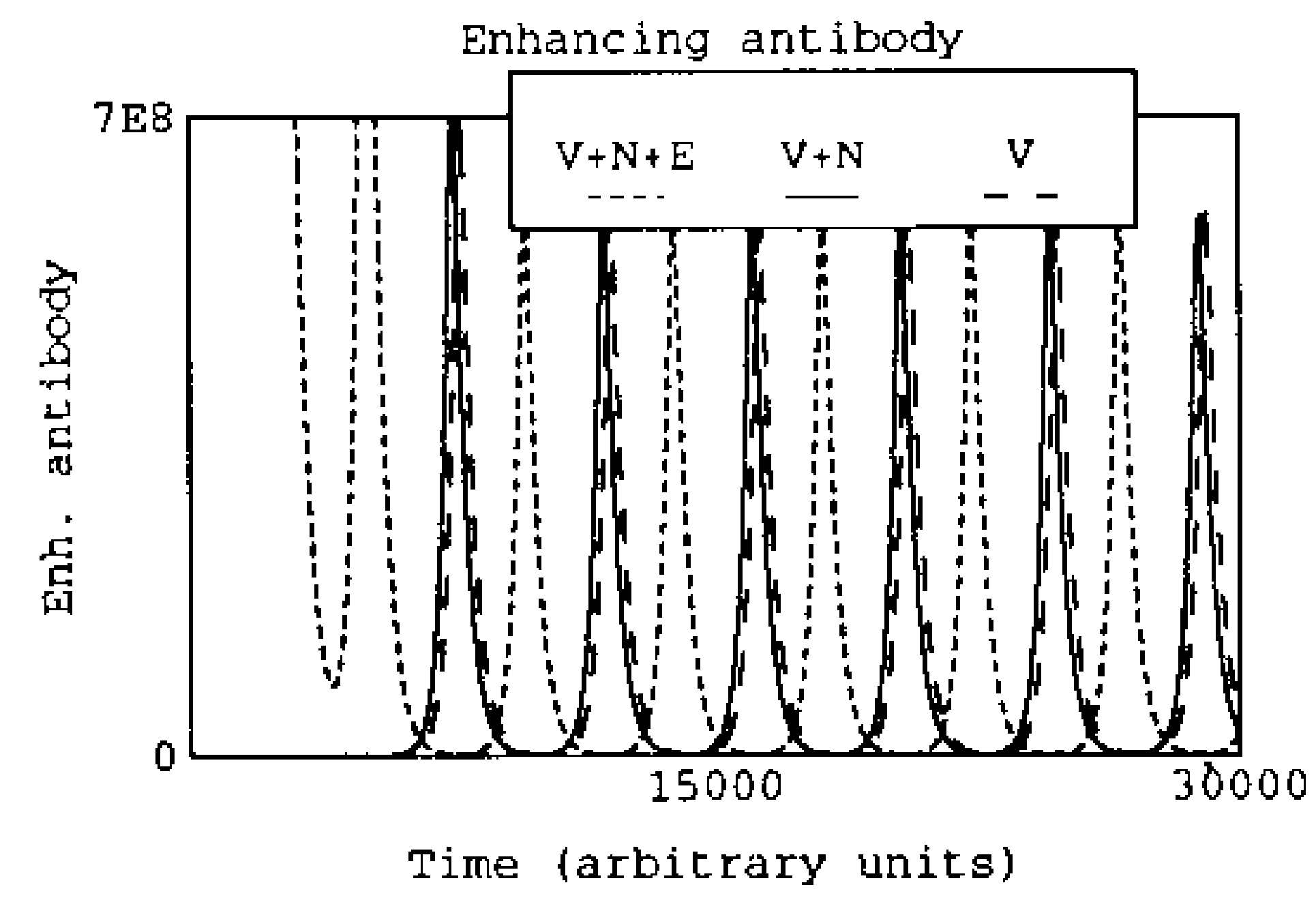

Fig. 9: enhancing antibody behavior after inoculation of virus antigen and neutralizing vaccine antigen $(V+$ $\mathrm{N})$, neutralizing and enhancing vaccine antigens $(\mathrm{V}+\mathrm{N}$ $+E$ ) and virus antigen alone (V). Vaccine antigen was inoculated at time zero and virus at time 1,500. The virus inoculum was 1,500 particles. Parameter values and initial values as in previous figures. Extreme values are not shown in figure.

Following vaccination with an antigen that induces both types of antibodies, the neutralizing antibodies tend to decrease until they are stimulated by the growing virus (Fig. 8). Since infection establishes earlier in the presence of enhancing antibodies, neutralizing antibodies induced by virus antigen also appear earlier $(V$ $+\mathrm{N}+\mathrm{E}$ ). When just a neutralizing vaccine is used $(V+N)$, the concentration of these anti- bodies follows almost the same course seen in a natural infection (V). The dynamics of enhancing antibodies (Fig. 9) follows a similar pattern, except that the initial concentration of these antibodies is zero when a neutralizing vaccine is used.

\section{DISCUSSION}

Perelson's equations "sketch the beginnings of a general model that can potentially account for many of the immunological consequences of HIV infection" (Perelson, 1989, p. 353). Starting from a modification of these equations we developed a model containing variables relevant to the simulation of immunological changes in the individual after a vaccination occurs. We introduced new variables and reexpressed old variables changing the predictive properties of the model.

Our modifications include the following:

1. introduction of additional compartments:

- a vaccine antigen compartment.

- a neutralizing antibody compartment.

- an infection enhancing antibody compartment.

2. additional viral properties:

- induction of susceptible T4 cell proliferation.

- activation of latently infected T4 cells.

- neutralization through binding to neutralizing antibodies.

- antibody-enhanced virus infection of susceptible T4 cells.

3. vaccine antigen interaction with the immune system:

- vaccine-induced susceptible T4 cell proliferation.

- vaccine-induced activation of latently infected T4 cells.

- vaccine stimulation of infection-enhancing antibodies.

- vaccine stimulation of neutralizing antibodies.

In addition to a viral antigen we also model a vaccine antigen compartment which shows a very simple dynamics. This approach allows us to investigate the sensitivity of the system to the initial dosage of the vaccine and the time constant of the exponential decay. The source of the different aspects of immunity (Table I) induced by a vaccine are coupled to just one compartment. The vaccine can have 
all the properties of the viral antigen except it cannot infect $\mathrm{T} 4$ cells or slow their growth rate. The properties can each be analyzed separately.

Virus and vaccine antigens stimulate $\mathrm{T} 4$ cell proliferation. This mimics an essential property of the immune system behavior missing in the simplified version of Perelson's general model (Perelson, 1989), but that is contained in other simple models (Cooper, 1986; Anderson, 1989). With most infections, an increase in T4 cell concentration activates various aspects of the immune protection. In the context of HIV infection, however, an increase in these cells also increases the number of host cells for virus infection. The equations proposed here help to understand the final balance determining whether infection will be established in the face of these opposing forces. The increase in the number of T4 cells after vaccination explains why viral infection establishes among vaccinated individuals earlier than among nonvaccinated ones even when the vaccine does not induce an enhancement of infection (Fig. 7). Thus a vaccine might have the undesirable effect of helping the virus overcome an otherwise protective immune response against HIV.

Virus and vaccine antigens also activate latently infected T4 cells. The mechanism is described as part of the physiopathology of HIV infection and suggested as a potential undesirable outcome of a vaccine (Levy, 1989; Rosenberg \& Fauci, 1989). This infection enhancing effect of the vaccine is to be distinguished from the enhancing antibodies described below. The effects can be uncoupled from one another in our simulations.

The vaccine antigen might stimulate the immune system in two directions. On one hand, neutralizing antibodies are produced which inactivate free viruses (Putney et al., 1986; Rusche et al., 1987). On the other hand, enhancing antibodies increase the biding rate of HIV to T4 cells by possibly facilitating cell attachment (Bolognesi, 1989a; Levy, 1989).

Our model is appropriate to study the equilibrium conditions and to analyze the sensitivity of a system that is governed by the final balance of at least four opposing biological ideas: antigen-stimulated increase in T4 cells; antigen-dependent activation of latent cells; neutralization of free virus; and antibody enhancement of infection.

The effect of vaccination strategies on the establishment and course of infection can be investigated in various ways. The timing of vaccination in relation to virus infection, and the dosage and frequency of revaccination could affect the efficacy of the vaccine in preventing infection and disease. Individuals can be vaccinated prior to virus inoculation, concomitant with infection or succeeding it at different stages of infection, as an immunotherapy. In all simulations presented here, vaccination precedes virus inoculation by the same time interval.

Our model can be extended in several different directions. We could include three more important antigen-specific $T$ cell subsets, such as $T_{v}$ for the $\mathrm{T}$ cells responding just to virus antigens, $T_{v a c}$ for the $T$ cells responding only to vaccine antigens, and $T_{e}$, representing $\mathrm{T}$ cells that respond to external challenges, tuberculosis for example. In the present formulation, $T_{v}$ and $T_{v a c}$ are combined in the same compartment, and there are no external sources disrupting the immune system equilibrium besides HIV and vaccine. Exposure to other infectious agents might change the course of HIV infection through activation of latent cells (Fauci, 1988; Smith et al., 1988; Rosenberg \& Fauci, 1989). Immune models incorporating T4 cells, HIV, and opportunistic infections have been shown to exhibit chaotic behavior (Anderson, 1989; Reibnegger et al., 1989). This could have profund consequences for the efficacy of a vaccine antigen in preventing disease.

One drawback of the model is that it lacks immune memory. This could be added by a compartment of $T$ cells or cells that produce antibodies that differentiate only after initial HIV activation, but that then continually reproduce.

In addition to the humoral responses represented in our equations, the cellular immunity is also active during HIV infection (Bolognesi, 1989a). We have not allowed for any of the protective mechanisms of cellular immunity in this model. In his general formulation of an AIDS immune model, Perelson (1989) points out several possibilities to include:

1. T cell growth as a function of antigen concentration (already present in our equa- 
tions), lymphokines and antigen presentation by macrophages.

2. Loss of uninfected T4 cells through autoimmune mechanisms. Various immune system effector functions need to be explicitly incorporated in the model: antibody and complement, antibody-dependent cellular cytotoxicity (ADCC) or cytotoxic T lymphocytes (CTL).

3. Loss of actively infected cells by mechanisms of cell mediated immunity (CTL, ADCC, natural killer cells, etc).

Virulence is assumed constant in the present formulation of the AIDS vaccine model. Biological evidence points to the fact that when antibodies to p24 start going down, virus density begins to increase (Weber et al., 1987). It is possible to represent an increase in virulence as time progresses by making virus release per active cell ( $N$ and $N_{s}$ ) explicit functions of time or of other proxy variables indicating changes in the level of immunological protection. For example, we could change $N$ and $N_{s}$ after an incubation period of 8 years or as a function of the level of antibodies to p24.

A final point relevant to modeling an AIDS vaccine concerns the relationship between the mode of transmission and the mechanism of vaccine action. Infection can be transmitted from person to person either as free or cellassociated virus, so both humoral and cellmediated immunity are of interest. In the preliminary results presented in this paper, transmission occurred only through inoculation of free virus. Antibodies as modeled here are likely to be the primary defense against an initial invasion by free virus. Other ways to challenge a previously immunized organism could be simulated through an increase in $T^{*}$, $T^{* *}$ or $S$ compartments directly. Each of these challenges might describe what most probably occurs in different epidemiological risk groups, e. g, IV drug users and homosexuals. Vaccines that block transmission by cell-to-cell contact, or stimulates elimination of infected cells would be especially relevant for these situations. Little is known about the fate of cell associated virus when transmitted to a susceptible host. It could have a crucial hole in determining the efficacy of an HIV vaccine. The use of models like the one described in this paper might help us to gain a better understanding of the effects of a vaccine on the natural history of the virus in a host.

\section{REFERENCES}

ANDERSON, R. M., 1989. Editorial review: Mathematjcal and statistical studies of the epidemiology of HIV. AIDS, 3: 333-346.

APTECH SYSTEMS, INC., 1988. The Gauss System version 2.0. Kent, Washington.

BOLOGNESI, D. P., 1989a. Prospects for prevention of and early intervention against $H I V . J A M A, 261$ : 3007-3013.

BOLOGNESI, D. P., 1989b. Do antibodies enhance the infection of cells by HIV? Nature, 340: 431-432.

COHEN, J, 1990. AIDS vaccine conference: Is "more" better? Science, 250: 369-370.

COOPER, L. N., 1986. Theory of an immune system retrovirus. Proc, Natl Acad. Sci. USA, 83; 91599163.

FAUCI, A. S., 1988. The human immunodeficiency virus: Infectivity and mechanisms of pathogenesis. Science, 239: 617-622.

FORD, M. P., 1988. SimGauss version 1.0. Forward Computing and Control Pty, Ltd, N. S. W. Australia.

KOFF, W. C., \& HOTH, D. F., 1988. Development and testing of AIDS vaccines, Science, 241: 426-432.

LEVY, J. A., 1988. Mysteries of HIV: challenges for therapy and prevention. Nature, 333: 519-522.

LEVY, J. A., 1989. Human immunodeficiency viruses and the pathogenesis of AIDS. JAMA, 261: 29973006.

PERELSON, A. S., 1989. Modeling the interaction of the immune system with HIV, p. 350-370. In C. CastilloChavez, Lecture Notes in Biomathematics 83. Mathematical and Statistical Approaches to AIDS Epidemiology. Springer-Verlag, Berlin.

PUTNEY, S. D.; MATHEWS, T. J.; ROBEY, W. G.; LYNN, D. L.; ROBERT-GUROFF, M.; MUELLER, W. T.; LANGLOIS, A. J.; GHRAYEB, J.; PETTEWAY, S. R.; WEINHOLD,K.J.; FISCHINGER, P. J.; WONG-STAAL, F.; GALLO, R. C. \& BOLOGNESI, D.P., 1986. HTLV-III/LAV-neutralizing antibodies to an e. coli-produced fragment of the virus envelope. Science, 234: 1392-95.

REIBNEGGER, G.; FUCHS, D.; HAUSEN, A.; WERNER, E. R.; WERNER-FELMAYER, G.; DIERJCH, M. P. \& WACHTER, H., 1989. Stability analysis of simple models for immune cells interacting with normal pathogens and immune system retroviruses. Proc. Nall Acad. Sci. USA, 86: 2026-2030.

ROSENBERG, Z. F. \& FAUCI, A. S., 1989. Minireview: Induction of expression of HIV in latently or chronically infected cells. AIDS Research and Human Retroviruses, 5:1-4,

RUSCHE, J. R.; LYNN, D. L.; GUROFF, M. R.; LANGLOIS, A. J.; LYERLY, H. K.; CARSON, H.; KROHN, K.; RANKI, A.; GALLO, R. C.; BOLOGNESI,D.P.\& MATTHEWS,T.J.; PARKER, D.; ROBERTS, C.; DUNCAN, J.; WELLER, I.; CARNE, C.; TEDDER, R. S.; PINCHING, A. J. \& CHEINGSONG-POPOV, R., 1987. Humoral immune response to the entire human immunodeficiency virus envelope glycoprotein made in insect cells. Proc. Natl Acad. Sci. USA, 84: 6924-28, 1987.

SCHAFFER, W.M.; TRUTY,G.L.\& FULMER, S., 1988. Dynamical Software. Dynamical Systems, Inc., Tucson, Arizona. 
SMITH, P. G.; MORROW, R. H. \& CHIN, J., 1988. Investigating interactions between HIV and tropical diseases. Int J. Epidemiol., 17: 705-707.

WEBER, J. N.; CLAPHAM, P. R. \& WEISS, R. A., 1987. Human immunodeficiency virus infection in two cohorts of homosexual men: neutralizing sera and association of anti-gag antibody with prognosis. Lancet, $1:$ 119-122.

WOLFRAM, S., 1988. Mathematica a system for doing mathematics by computer. Addison-Wesley Publishing Company, New York. 